IOANNis K. Argyros (Lawton, OK)

\title{
ON THE GAP BETWEEN THE SEMILOCAL CONVERGENCE DOMAINS OF TWO NEWTON METHODS
}

Abstract. We answer a question posed by Cianciaruso and De Pascale: What is the exact size of the gap between the semilocal convergence domains of the Newton and the modified Newton method? In particular, is it possible to close it? Our answer is yes in some cases. Using some ideas of ours and more precise error estimates we provide a semilocal convergence analysis for both methods with the following advantages over earlier approaches: weaker hypotheses; finer error bounds on the distances involved, and at least as precise information on the location of the solution; and a smaller gap between the two methods.

1. Introduction. In this study we are concerned with the problem of approximating a locally unique solution of the nonlinear equation

$$
F(x)=0,
$$

where $F$ is a Fréchet differentiable operator defined on a convex subset $D$ of a Banach space $X$ with values in a Banach space $Y$.

The most popular methods for generating a sequence $\left\{x_{n}\right\}(n \geq 0)$ approximating a solution of (1.1) are Newton's method

$$
x_{n+1}=x_{n}-F^{\prime}\left(x_{n}\right)^{-1} F\left(x_{n}\right) \quad\left(n \geq 0, x_{0} \in D\right),
$$

and the modified Newton's method

$$
x_{n+1}=x_{n}-F^{\prime}\left(x_{0}\right)^{-1} F\left(x_{n}\right) \quad(n \geq 0) .
$$

There is an extensive literature on local as well as semilocal convergence results for both methods under various assumptions (see [1]-[4], [8], [9], and the references there).

2000 Mathematics Subject Classification: 65H10, 65G99, 47H17, 49M15.

Key words and phrases: Newton's method, modified Newton's method, Banach space, Hölder continuity, semilocal convergence, regular smoothness, Vertgeim condition, convergence domain. 
We are motivated by the elegant work by A. Galperin [7] dealing with the question posed in [4]: What is the exact size of the gap between sufficient convergence conditions for methods (1.2) and (1.3)

Here is what we know: Let $F_{0}=F^{\prime}\left(x_{0}\right)^{-1} F$ be the normalized operator for $F$.

- B. A. Vertgeim [10]: if $F_{0}^{\prime}$ is $\lambda$-Hölder continuous on $D(\lambda \in(0,1])$, i.e.

$$
\left\|F_{0}^{\prime}(x)-F_{0}^{\prime}(y)\right\| \leq l\|x-y\|^{\lambda}
$$

for all $x, y \in D$, then method (1.3) converges to $x^{*}$ provided that

$$
h=l\left\|F_{0}\left(x_{0}\right)\right\|^{\lambda} \leq\left(\frac{\lambda}{1+\lambda}\right)^{\lambda}
$$

whereas Newton's method converges provided that

$$
h \leq V_{\lambda}\left(\frac{\lambda}{1+\lambda}\right)^{\lambda}
$$

where $V_{\lambda}$ is the unique solution of the equation

$$
t^{\frac{\lambda}{1+\lambda}}+\lambda^{\frac{\lambda}{1+\lambda}} t=\left(\frac{1+\lambda}{\lambda^{\frac{\lambda}{1+\lambda}}}\right)^{\lambda}
$$

- F. Cianciaruso and E. De Pascale [4]: the $V_{\lambda}$ can be replaced by an at least as large parameter $C_{\lambda}$ such that

$$
h \leq C_{\lambda}\left(\frac{\lambda}{1+\lambda}\right)^{\lambda}
$$

which is the reciprocal of the number

$$
\alpha(\lambda)=\min \left\{b \geq 1: \max _{0 \leq t \leq t(\lambda)} g(t) \leq b\right\},
$$

where

$$
g(t)=\frac{t^{1+\lambda}+(1+\lambda)^{t}}{(1+t)^{1+\lambda}-1}
$$

- A. Galperin [7]: The $C_{\lambda}$ can be replaced by an at least as large parameter $G_{\lambda}$ given by

$$
G_{\lambda}=\left[\frac{1+\lambda}{\lambda} \lim _{s \rightarrow \infty} y_{n}(0)\right]^{\lambda},
$$

where for $y_{0}(s)=1-s, y_{n+1}(s)$ is the unique solution of the equation (with unknown $r$ )

$$
\frac{r^{1+\lambda}}{(1+\lambda)\left[1-(s+r)^{\lambda}\right]}=y_{n}(s+r) .
$$

In fact the following table was given in [7]. 
Table 1. Comparison table

\begin{tabular}{ccccccccccc}
\hline$\lambda$ & .1 & .2 & .3 & .4 & .5 & .6 & .7 & .8 & .9 & 1 \\
$\lambda V_{\lambda}$ & .204 & .339 & .453 & .553 & .644 & .727 & .803 & .873 & .939 & 1 \\
$C_{\lambda}$ & .542 & .590 & .640 & .692 & .746 & .799 & .852 & .903 & .952 & 1 \\
$G_{\lambda}$ & .605 & .686 & .748 & .801 & .846 & .885 & .920 & .950 & .976 & 1 \\
\hline
\end{tabular}

In view of the above table Galperin concluded that the gap between the convergence domains of methods (1.2) and (1.3) is positive and cannot be closed under condition (1.4). Moreover, he showed that this is also the case under the $\omega$-smoothness assumption:

$$
\left\|F_{0}(x)-F_{0}(y)\right\| \leq \omega(\|x-y\|) \quad \text { for all } x, y \in D
$$

where $\omega$ is a nonzero nondecreasing concave function on $[0, \infty)$ with $\omega(0)=0$. Note that a possible choice of $\omega$ is

$$
\omega(t)=l t^{\lambda} .
$$

Furthermore, under the regular smoothness condition:

$$
\begin{aligned}
\omega^{-1}[\min \{\|A(x)\|,\|A(y)\|\}+\|A(x)-A(y)\|] \\
-\omega^{-1}[\min \{\|A(x)\|,\|A(y)\|\}] \leq\|x-y\|
\end{aligned}
$$

for $A: D \rightarrow L(X, Y), x$ fixed in $D$ and all $y \in D$, Galperin showed that the gap can be closed for $A=F_{0}^{\prime}$.

In the next two sections we provide our contributions with the advantages as already stated in the abstract.

2. Semilocal convergence of methods (1.2) and (1.3). Let us introduce the center $\lambda$-Hölder condition

$$
\left\|F_{0}^{\prime}(x)-I\right\| \leq l_{0}\left\|x-x_{0}\right\|^{\lambda} \quad \text { for all } x \in D
$$

and set

$$
h_{0}=l_{0}\left\|F_{0}\left(x_{0}\right)\right\|^{\lambda} \leq\left(\frac{\lambda}{1+\lambda}\right)^{\lambda} .
$$

Clearly

$$
l_{0} \leq l
$$

and $l / l_{0}$ may be arbitrarily large [1], [3]. Note that

$$
h \leq\left(\frac{\lambda}{1+\lambda}\right)^{\lambda} \Rightarrow h_{0} \leq\left(\frac{\lambda}{1+\lambda}\right)^{\lambda},
$$

but not vice versa unless $l_{0}=l$. In [2] we showed that (2.1) and (2.2) can replace (1.4) and (1.5) respectively in the study of the convergence of method (1.3) with the following advantages: 
(A) (1) weaker hypotheses;

(2) finer error bounds on the distances involved;

(3) an at least as precise information on the location of the solution;

(4) smaller gap between methods (1.2) and (1.3).

In the case of method (1.2) we showed that if we use the combination of conditions (1.4) and (2.1), instead of only (1.4), the condition corresponding to $(1.5)$ is given for $l_{0}=l d, d \in[0,1]$, by

$$
h \leq\left\{\begin{aligned}
{\left[1+\delta d /(1-q)^{\lambda}\right]^{-1} \delta, \delta=(1+\lambda) q, \lambda \in[0,1) } & \\
& \text { for some } q \in[0,1) \text { and } \eta, \lambda \\
& \text { not zero at the same time, } \\
(1-\bar{\delta} d)^{-1} d \quad & \text { for some } \bar{\delta} \in[0,1] \text { and } \lambda=1
\end{aligned}\right.
$$

In particular, if we take $\lambda=1$ (Lipschitz case) and $\bar{\delta}=1$ conditions (1.5), (2.5) become

$$
h_{k}=l\left\|F_{0}\left(x_{0}\right)\right\| \leq \frac{1}{2},
$$

and

$$
h_{A}=\bar{l}\left\|F_{0}\left(x_{0}\right)\right\| \leq \frac{1}{2}, \quad \bar{l}=\frac{l+l_{0}}{2},
$$

respectively. Note that (2.6) is the famous Newton-Kantorovich hypothesis [9] which is a sufficient convergence condition for Newton's method (1.2) in the Lipschitz case. Note again that

$$
h_{k} \leq \frac{1}{2} \Rightarrow h_{A} \leq \frac{1}{2}
$$

but not vice versa unless $l_{0}=l$. For $\lambda \in[0,1)$ and

$$
q=\frac{1}{1+\lambda}
$$

in view of condition (2.5) we get

$$
h \leq A_{\lambda}\left(\frac{\lambda}{1+\lambda}\right)^{\lambda}
$$

where

$$
A_{\lambda}=\left[d+\left(\frac{\lambda}{1+\lambda}\right)^{\lambda}\right]^{-1} .
$$

Using e.g. $d=1 / 2$ we obtain the table:

Table 2. Comparison table

\begin{tabular}{ccccccccccc}
\hline$\lambda$ & .1 & .2 & .3 & .4 & .5 & .6 & .7 & .8 & .9 & 1 \\
$G_{\lambda}$ & .605 & .686 & .748 & .801 & .846 & .885 & .920 & .950 & .976 & 1 \\
$A_{\lambda}$ & .777 & .833 & .874 & .904 & .926 & .948 & .964 & .978 & .990 & 1 \\
\hline
\end{tabular}


It shows that the gap between the convergence domains gets even smaller than in all previous works. In fact it can be closed completely: We note that since $l / l_{0}$ (i.e., $\left.1 / d\right)$ is arbitrarily large there exists a unique $\lambda \in[0,1]$ such that

$$
d+\left(\frac{\lambda}{1+\lambda}\right)^{\lambda}=1
$$

i.e.

$$
A_{\lambda}=1 \text {. }
$$

That is, taking into account the ratio $l / l_{0}$ one can sometimes find a $\lambda \in[0,1]$ such that the gap is zero.

To further compare our approach with the one in [7], we assume that the normalized operator $F_{0}$ is $\omega_{0}$-smooth on $D$ relative to $x_{0} \in D$ :

$$
\left\|F_{0}^{\prime}(x)-F_{0}^{\prime}\left(x_{0}\right)\right\| \leq \omega_{0}\left(\left\|x-x_{0}\right\|\right) \quad \text { for all } x \in D,
$$

where the function $\omega_{0}$ is as $\omega$.

Note that

$$
\omega_{0}(t) \leq \omega(t) \quad \text { for all } t \in[0, \infty)
$$

and $\omega / \omega_{0}$ may be arbitrarily large [1], [3].

If we set

$$
\omega_{0}(t)=l_{0} t^{\lambda}
$$

then condition (2.14) reduces to (2.1), whereas if $l_{0}=l$ then $\omega_{0}(t)=\omega(t)$ for all $t \in[0, \infty)$.

Let us introduce the notation

$$
\bar{t}_{n}=\left\|x_{n}-x_{0}\right\|, \quad \bar{\varepsilon}_{n}=\left\|x_{n+1}-x_{n}\right\| .
$$

Define

$$
\Omega_{0}(t)=\int_{0}^{t} \omega_{0}(r) d r
$$

and

$$
\Omega(t)=\int_{0}^{t} \omega(r) d r .
$$

Consider the map $P: \mathbb{R}_{+}^{2} \rightarrow \mathbb{R}_{+}^{2}$ that maps each nonnegative pair $(t, \omega)$ to $\left(t_{+}, \varepsilon_{+}\right)$according to the formulas

$$
t_{+}=t+\varepsilon, \quad \varepsilon_{+}=\Omega_{0}(t+\varepsilon)-\Omega_{0}(t)
$$

in the case of method (1.3) and

$$
t_{+}=t+\varepsilon, \quad \varepsilon_{+}=\Omega(t+\varepsilon)-\Omega(t)
$$

in the case of method (1.2). 
Finally, define functions $f_{0}$ and $f$ on $[0, \infty)$ by

$$
f_{0}(t)=\Omega_{0}(t)-t+\varepsilon_{0},
$$

and

$$
f(t)=\Omega(t)-t+\varepsilon_{0} .
$$

Then by simply replacing $\omega, \Omega$ by $\omega_{0}, \Omega_{0}$ respectively in Theorem 2.3 of $[7$, p. 389], we obtain the following semilocal convergence theorem for method (1.3):

TheOREM 2.1. Assume that the normalized operator $F_{0}$ is $\omega_{0}$-smooth on $D$ relative to $x_{0} \in D$, and

$$
\left\|F_{0}\left(x_{0}\right)\right\| \leq \varepsilon_{0} \leq \alpha_{0}-\Omega_{0}\left(\alpha_{0}\right), \quad \alpha_{0}=\omega_{0}^{-1}(1) .
$$

Then:

(a) The sequence $\left\{t_{n}\right\}$ generated by $(2.20)$ and starting from $\left(0, \varepsilon_{0}\right)$ converges to $t^{*}$, the smaller of the two zeros of function (2.22). Moreover, for all $n \geq 0$,

$$
\begin{aligned}
& \bar{t}_{n} \leq t_{n}, \\
& \bar{\varepsilon}_{n} \leq \varepsilon_{n} .
\end{aligned}
$$

(b) The sequence $\left\{x_{n}\right\}$ generated by method (1.3) is well defined, remains in the ball $U\left(x_{0}, t_{\infty}\right)=\left\{x \in X:\left\|x-x_{0}\right\| \leq t_{\infty}\right\}, t_{\infty}=\lim _{n \rightarrow \infty} t_{n}$, and converges to a solution $x_{\infty}$ of equation (1.1). Moreover, for all $n \geq 0$,

$$
\begin{aligned}
\left\|x_{\infty}-x_{n}\right\| & \leq t_{\infty}-t_{n}, \\
\left\|F_{0}\left(x_{n}\right)\right\| & \leq \varepsilon_{n} .
\end{aligned}
$$

(c) The solution $x_{\infty}$ is unique in $U\left(x_{0}, t^{* *}\right)$, where $t^{* *}$ is the larger of the two zeros of $f_{0}$.

(d) The convergence condition (2.24), the radii $r^{*}, t^{* *}$, and the bounds (2.27) and (2.28) are sharp: they are attained for the function $F_{0}$ which is $\omega_{0}$-smooth on $[0, \infty)$.

REMARK 2.2. If equality holds in (2.15) our Theorem 2.1 reduces to Theorem 2.3 of [7]. Otherwise, the advantages (A) mentioned above of our approach over the one in [7] hold true.

In the case of method (1.2), retaining the notation introduced above we have:

LEMMA 2.3. Under conditions (1.13) and (2.14) the following estimates hold for method (1.2):

$$
\bar{t}_{n+1} \leq \bar{t}_{n}+\bar{\varepsilon}_{n}
$$


and

$$
\bar{\varepsilon}_{n+1} \leq \frac{\Omega\left(\bar{\varepsilon}_{n}\right)}{1-\omega_{0}\left(\bar{t}_{n}+\bar{\varepsilon}_{n}\right)} \quad \text { for all } n \geq 0 .
$$

Proof. In view of (2.14) we obtain

$$
\left\|F_{0}^{\prime}\left(x_{n+1}\right)-F_{0}^{\prime}\left(x_{0}\right)\right\| \leq \omega_{0}\left(\left\|x_{n+1}-x_{0}\right\|\right) \leq \omega_{0}\left(\bar{t}_{n+1}\right)<1 .
$$

Using (2.31) and the Banach lemma on invertible operators [9] we obtain $F_{0}^{\prime}\left(x_{n+1}\right)^{-1} \in L(Y, X)$ and

$$
\left\|F_{0}^{\prime}\left(x_{n+1}\right)^{-1}\right\| \leq \frac{1}{1-\left\|F_{0}^{\prime}\left(x_{n+1}\right)-F_{0}^{\prime}\left(x_{0}\right)\right\|} \leq \frac{1}{1-\omega_{0}\left(\bar{t}_{n+1}\right)} .
$$

The rest follows exactly as in Lemma 3.1 of [7].

REMARK 2.4. If equality holds in (2.15) our Lemma 2.3 reduces to Lemma 3.1 of [7]. Otherwise it is an improvement since our $\left(\bar{t}_{n}, \bar{\varepsilon}_{n}\right)$ are smaller than the corresponding ones in [7] (obtained by simply replacing $\omega_{0}$ by $\omega$ in $(2.30))$.

Define the map $p: \mathbb{R}^{2} \supset \operatorname{Dom} p \rightarrow \mathbb{R}^{2}$ sending any pair in

$$
\operatorname{Dom} p=\left\{(t, \varepsilon) \mid t \geq 0, \varepsilon \geq 0, t+\varepsilon \leq \alpha_{0}\right\}
$$

to $\left(t_{+}, \varepsilon_{+}\right)$where

$$
t_{+}=t+\varepsilon, \quad \varepsilon_{+}= \begin{cases}\frac{\Omega(\varepsilon)}{1-\omega_{0}(t+\varepsilon)}, & t+\varepsilon<\alpha_{0}, \\ 0, & t+\varepsilon=\alpha_{0} .\end{cases}
$$

As in Theorem 4.1 of [6] the convergence domain $U=U_{\left(l_{0}, l\right)}=\left\{\left(t_{0}, \varepsilon_{0}\right)\right.$ $\left.t_{n}<\alpha_{0}\right\}$ of $(2.34)$ is given by

$$
U=\left\{\left(t_{0}, \varepsilon_{0}\right) \mid 0 \leq t_{0} \leq \alpha_{0} \text { and } 0 \leq \varepsilon_{0} \leq \chi\left(t_{0}, \alpha_{0}\right)\right\}
$$

where $\chi\left(t, t^{\prime}\right)$ is the unique nonzero and nonincreasing solution of

$$
\frac{\Omega(z(t))}{1-\omega_{0}[t+z(t)]}=z(t+z(t)), \quad z\left(t^{\prime}\right)=0 .
$$

For $\omega_{0}, \omega$ fixed we can compose the functions $\varepsilon=\chi\left(t, t^{\prime}\right)$ and $t^{\prime}=I(t, \varepsilon)$, where $I$ is the implicit function defined by $\chi\left(t, t^{\prime}\right)=\varepsilon$. In particular we can compute $\chi\left(0, \alpha_{0}\right)$.

According to Theorem 4.3 of [6], $\chi\left(t, t^{\prime}\right)=\lim _{n \rightarrow \infty} z_{n}(t)$, where $z_{0}(t)=$ $t^{\prime}-t$, and $z_{n+1}(t)$ is the unique solution of the equation

$$
\frac{\Omega(\varepsilon)}{1-\omega_{0}(t+\varepsilon)}=z_{n}(t+\varepsilon)
$$

for $\varepsilon \in\left[0, z_{n}(t)\right]$.

We can state the following semilocal convergence theorem for Newton's method (1.2): 
THEOREM 2.5. Suppose that the normalized operator $F_{0}$ is $\omega_{0}$-smooth relative to $x_{0} \in D$ and $\omega$-smooth on $D$. Moreover, assume that

$$
\left\|F_{0}\left(x_{0}\right)\right\| \leq \varepsilon_{0} \leq \chi\left(0, \alpha_{0}\right)
$$

Then

(a) The scalar sequence $\left\{t_{n}\right\}$ generated by (2.34) starting at $\left(0, \varepsilon_{0}\right)$ converges to the zero of the function $t \mapsto \chi\left[t, I\left(0, \varepsilon_{0}\right)\right]$, and for all $n \geq 0$,

$$
\bar{t}_{n} \leq t_{n} \quad \text { and } \quad \bar{\varepsilon}_{n} \leq \varepsilon_{n} .
$$

(b) The sequence $\left\{x_{n}\right\}$ generated by Newton's method (1.2) is well defined, remains in $U\left(x_{0}, t_{\infty}\right)$ for all $n \geq 0$ and converges to a unique solution of the equation $F(x)=0$ in $U\left(x_{0}, t^{* *}\right)$, where $t^{* *}$ is the greatest zero of $f$. Moreover, for all $n \geq 0$,

$$
\begin{aligned}
\left\|x_{\infty}-x_{n}\right\| & \leq t_{\infty}-t_{n}, \\
\left\|F_{0}\left(x_{n+1}\right)\right\| & \leq \Omega\left(\varepsilon_{n}\right) .
\end{aligned}
$$

Proof. Use estimate (2.32), (2.34) instead of the corresponding estimates

$$
\left\|F_{0}^{\prime}\left(x_{n+1}\right)\right\|^{-1} \leq \frac{1}{1-\omega\left(\bar{t}_{n+1}\right)}
$$

and (2.34) for $\omega_{0}=\omega$ used in [7]. The rest of the proof follows exactly as in Theorem 3.2 in [7, p. 393].

REMARK 2.6. If equality holds in (2.15) our Theorem 2.5 reduces to Theorem 3.2 of [7]. Otherwise it is an improvement with the advantages (A) mentioned above.

If the function $\omega_{0}$ is given by (2.16), then the equation (2.37) becomes

$$
\frac{l \varepsilon^{1+\lambda}}{(1+\lambda)\left[1-d l(t+\varepsilon)^{\lambda}\right]}=z_{n}(t+\varepsilon) .
$$

Then we can show exactly as in Proposition 3.3 in [7]:

Proposition 2.7. For all $l>0$,

$$
l^{1 / \lambda} \chi\left(0, l^{-1 / \lambda}\right)=\lim _{n \rightarrow \infty} y_{n}(0),
$$

where $y_{0}(s)=1-s$, and $y_{n+1}(s)$ is the unique solution of the equation

$$
\frac{r^{1+\lambda}}{(1+\lambda)\left[1-d(s+r)^{\lambda}\right]}=y_{n}(s+r)
$$

and

$$
\bar{A}_{\lambda}=l \chi\left(0, l^{1 / \lambda}\right)\left(\frac{\lambda}{1+\lambda}\right)^{\lambda}=\left[\frac{1+\lambda}{\lambda} \lim _{n \rightarrow \infty} y_{n}(0)\right]^{\lambda}
$$


REMARK 2.8. If equality holds in (2.15) then Proposition 2.7 reduces to Proposition 3.3 in [7], and $G_{\lambda}=\bar{A}_{\lambda}$. Otherwise we have

$$
G_{\lambda} \leq \bar{A}_{\lambda}
$$

which improves the most recent estimate given by Galperin [7].

3. Semilocal convergence under regular smoothness. We refer the reader to [6]-[8] for the advantages of regular smoothness.

Denote by $N$ the class of nondecreasing functions $\omega:[0, \infty) \rightarrow[0, \infty)$ that are concave and vanishing at 0 . Given an $\omega \in N$, we say that $A: D \rightarrow$ $L(X, Y)$ is $\omega$-regularly continuous on $D$ relative to $x \in D$ or, equivalently, that $\omega$ is a regular continuity modulus of $A$ on $D$ relative to $X$, if

$$
\begin{aligned}
\omega^{-1}[\min \{\|A(x)\|,\|A(y)\|\} & +\|A(y)-A(x)\|] \\
& -\omega^{-1}(\min \{\|A(x)\|,\|A(y)\|\}) \leq\|y-x\|
\end{aligned}
$$

for all $y \in D$.

$A$ is $\omega$-regularly continuous on $D$ if the above is valid for all $x, y \in D$. The operator $F$ is $\omega$-regularly smooth on $D$ if its derivative $F^{\prime}$ is $\omega$-regularly continuous there. The function $\omega$ is then called a regular smoothness modulus of $F$ on $D$. A function is called regularly smooth on $D$ if it has a regular smoothness modulus on $D$.

Given $\omega_{0} \in N$, define functions $\psi$ and $\Psi$ by

$$
\begin{aligned}
\psi_{0}(u, t) & =\omega_{0}\left[(u-t)^{+}+t\right]-\omega_{0}\left[(u, t)^{+}\right] \\
& = \begin{cases}\omega_{0}(u)-\omega_{0}(u-t), & t \in[0, u], u \geq 0, \\
\omega_{0}(t), & t \geq u \geq 0,\end{cases} \\
\Psi(u, t) & =\int_{0}^{t} \psi(u, \tau) d \tau,
\end{aligned}
$$

where for a real number $a$,

$$
a^{+}=\max \{a, 0\} .
$$

We also need to introduce the generator

$$
t_{+}=t+\varepsilon, \quad \varepsilon_{+}=\Psi\left(\alpha_{0}, t+\varepsilon\right)-\Psi\left(\alpha_{0}, t\right)
$$

and the function

$$
\Phi(t)=\Psi\left(\alpha_{0}, t\right)-t+\varepsilon_{0}, \quad t \geq 0 .
$$

By simply replacing $\omega$ by $\omega_{0}$ in the proof of Theorem 5.2 in [7, p. 400] we can show the following semilocal theorem for method (1.3):

THEOREM 3.1. Suppose that $\omega_{0}$ is a regular continuity modulus of $F_{0}$ on $D$ relative to $x_{0}$. If

$$
\left\|F_{0}\left(x_{0}\right)\right\| \leq \varepsilon_{0} \leq \Omega\left(\alpha_{0}\right)
$$


then:

(a) The scalar sequence $\left\{t_{n}\right\}$ starting from $\left(0, \varepsilon_{0}\right)$ and generated by (3.5) converges to $t^{*}$, the smaller of the two zeros of $\Phi$, and for all $n \geq 0$,

$$
\bar{t}_{n} \leq t_{n} \quad \text { and } \quad \bar{\varepsilon}_{0} \leq \varepsilon_{n} .
$$

(b) The sequence $\left\{x_{n}\right\}$ generated by modified Newton method (1.3) is well defined, remains in $U\left(x_{0}, t^{*}\right)$ for all $n \geq 0$ and converges to a unique solution $x_{\infty}$ of equation (1.1) in $U\left(x_{0}, t^{* *}\right)$, where $t^{* *}$ is the largest zero of $\Phi$ :

$$
\begin{aligned}
\left\|x_{\infty}-x_{n}\right\| & \leq t^{*}-t_{n}, \\
\left\|F_{0}\left(x_{n}\right)\right\| & \leq \varepsilon_{n} .
\end{aligned}
$$

The convergence condition (3.7), the radius $t^{*}$, and the bounds (3.9) and (3.10) are sharp: they are attained for a function $\Phi$ which is $\omega_{0}$-regularly smooth on $\left[0, \alpha_{0}\right]$.

From now on we set

$$
\bar{\alpha}_{n}=\omega^{-1}\left(\left\|F_{0}^{\prime}\left(x_{n}\right)\right\|\right)
$$

and assume

(c) $\omega$ is a regular continuity modulus of $F_{0}$ on $D$ and $\omega_{0}$ is a regular continuity modulus of $F_{0}$ on $D$ relative to $x_{0}$.

Then as in Lemma 2.3 above and Lemma 6.1 in [7] we show:

LEMMA 3.2. Under the above stated hypothesis (c) we have

$$
\begin{aligned}
\bar{t}_{n+1} & \leq \bar{t}_{n}+\bar{\varepsilon}_{n}, \\
\bar{\alpha}_{n+1} & \geq\left(\bar{\alpha}_{n}-\bar{\varepsilon}_{n}\right)^{+}, \\
\bar{\delta}_{n+1} & \leq \frac{\Psi\left(\bar{\alpha}_{n}, \bar{\varepsilon}_{n}\right)}{1-\omega_{0}\left(\bar{\alpha}_{n+1}+\bar{t}_{n+1}\right)-\omega_{0}\left(\bar{\alpha}_{n+1}\right)} .
\end{aligned}
$$

Let us define the generator:

$$
t_{+}=t+\varepsilon, \quad \alpha_{+}=(\alpha-\varepsilon)^{+}, \quad \varepsilon_{+}=\frac{\Psi(\alpha, \varepsilon)}{1-\omega_{0}\left(\alpha_{+}+t_{+}\right)-\omega_{0}\left(\alpha_{+}\right)} .
$$

Then exactly as in Theorem 6.2 in [7] we can show the semilocal convergence result for Newton's method $(1.2)$ under $\left(\bar{\omega}_{0}, \omega\right)$ regular smoothness.

THEOREM 3.3. Under hypothesis (c), further assume that

$$
\left\|F_{0}\left(x_{0}\right)\right\| \leq \varepsilon_{0} \leq \Omega\left(\alpha_{0}\right) .
$$

Then: 
(a) The sequence $\left\{t_{n}\right\}$ generated by (3.16) starting at $\left(0, \varepsilon_{0}\right)$ converges to $t^{*}$, the smaller of the two zeros of $\Phi$ given by (3.6) (for $\left.\omega_{0}=\omega\right)$, and for all $n \geq 0$,

$$
\bar{t}_{n} \leq t_{n} \quad \text { and } \quad \bar{\varepsilon}_{n} \leq \varepsilon_{n} .
$$

(b) The sequence $\left\{x_{n}\right\}$ generated by Newton's method (1.2) is well defined, remains in $U\left(x_{0}, t^{*}\right)$ for all $n \geq 0$, and converges to a unique solution $x_{\infty}$ of equation (1.1) in $U\left(x_{0}, t^{* *}\right)$, where $t^{* *}$ is the largest of the two zeros of $\Phi$. Moreover, for all $n \geq 0$,

$$
\begin{aligned}
\left\|x_{\infty}-x_{n}\right\| & \leq t_{*}-t_{n}, \\
\left\|F_{0}\left(x_{n+1}\right)\right\| & \leq \bar{\varepsilon}_{n},
\end{aligned}
$$

where

$$
\bar{\varepsilon}_{n}=\varepsilon_{n} \omega_{0}\left(\alpha_{0}-t_{n}\right)-\Omega\left(\alpha_{0}-t_{n}\right)+\Omega\left(\alpha_{0}-t_{n+1}\right) .
$$

(c) The convergence condition (3.16), the radius $t^{*}$, and the bounds (3.18) and (3.19) are sharp: they are attained for the function $\Phi$.

REMARK 3.4. If $\omega_{0}=\omega$ Theorem 3.1 and Lemmas 3.1-3.2 reduce to Theorem 5.2 and Lemmas 6.1-6.2 of [7] respectively. Otherwise they constitute an improvement with the advantages as stated in the abstract.

\section{References}

[1] I. K. Argyros, A unifying local-semilocal convergence analysis and applications for two-point Newton-like methods in Banach space, J. Math. Anal. Appl. 298 (2004), 374-397.

[2] - Concerning the "terra incognita" between convergence regions of two Newton methods, Nonlinear Anal. 62 (2005), 179-194.

[3] - Approximate Solution of Operator Equations with Applications, World Sci., Hackensack, NJ, 2005.

[4] F. Cianciaruso and E. De Pascale, Newton-Kantorovich aproximations when the derivative is Hölderian: Old and new results, Numer. Funct. Anal. Optim. 24 (2003), $713-723$.

[5] J. A. Ezquerro, J. M. Gutiérrez, M. A. Hernández and M. A. Salanova, Solving nonlinear integral equations arising in radiative transfer, Numer. Funct. Anal. Optim. 20 (1999), 661-673.

[6] A. Galperin, Kantorovich majorization and functional equations, ibid. 24 (2003), 783-811.

[7] - On convergence domains of Newton's and modified Newton methods, ibid. 26 (2005), 385-405.

[8] A. Galperin and Z. Waksman, Newton-type methods under regular smoothness, ibid. 17 (1996), 259-291.

[9] L. V. Kantorovich and G. P. Akilov, Functional Analysis in Normed Spaces, Pergamon Press, Oxford, 1982. 
[10] B. A. Vertgeim, On conditions of applicability of Newton's method, Dokl. Akad. Nauk SSSR 110 (1956), 719-722 (in Russian).

Department of Mathematical Sciences

Cameron University

Lawton, OK 73505, U.S.A.

E-mail: iargyros@cameron.edu

Received on 26.10.2006;

revised version on 2.4.2007 